\title{
Computational Geometry and Spatial Meshes
}

\author{
César Otero, Reinaldo Togores \\ Dept. of Geographical Engineering and Graphical Expression Techniques \\ Civil Engineering School, University of Cantabria, Avda. de los Castros, s/n, \\ E-39005, Santander, Spain \\ oteroc@unican.es
}

\begin{abstract}
This paper establishes a relation between the field of study of Geometric Design of the Structures known as "Spatial Meshes" and Computational Geometry. According to some properties of Voronoi Diagrams and Convex Hulls, and by means of two transformations: one inversive, the other projective, a way for generating polyhedra approximating spheres and other second order surfaces (ellipsoids, paraboloids and hyperboloids), starting from a simple Voronoi Diagram, is shown. The paper concludes with the geometric design of C-TANGENT domes: a new way for generating Spatial Meshes that can become a possible field of application for Computational Geometry.
\end{abstract}

\section{Introduction: What is a Spatial Mesh?}
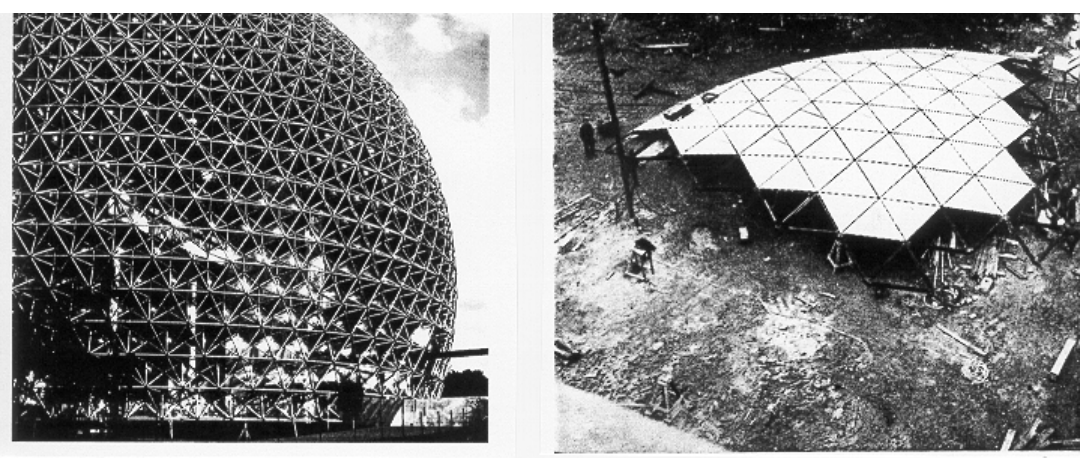

Fig. 1. Spatial structures: (left) spatial mesh; (right) plate structure

Structural design and calculus procedures can be made in a bi- or threedimensional fashion. Structures like those in Fig. 1 do not have a dominant bi-dimensional stress-resisting element; stresses are transmitted along the whole structure in such a way that it is impossible to study the isolated parts. Its unitary character prevails during the calculus and design process in this kind of structures. What marks a difference between them is their macroscopic character, 

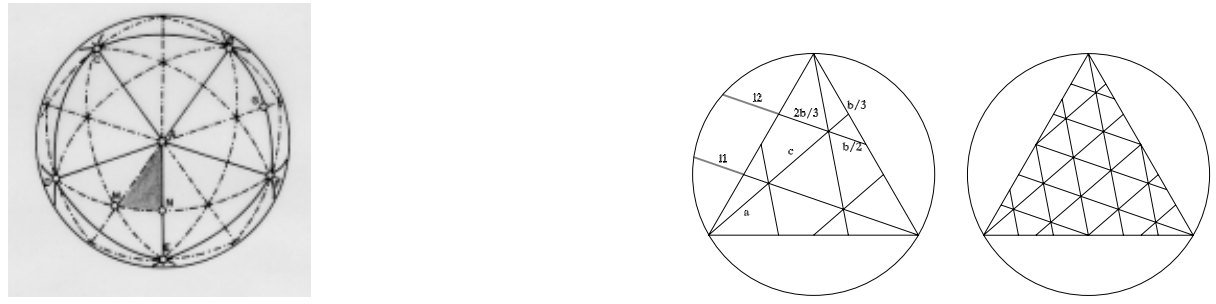

Fig. 2. (left) A geodesic mesh derived from a regular isosahedron; (right) two tesselations of the $\{3,5+\}_{b, c}$ type, proposed into a triangle

discrete in the case of the first illustration (Fig. 1-left) and continuous in the second one (Fig. 1-right). Both are Spatial Structures [3,4]; the first one of the Spatial Mesh type, the second one of Plate type. By definition [7] a Spatial Mesh is a structural system composed by linear elements, joined in such a way that stresses are transmitted in a tridimensional manner. In some cases, part of the component elements can be bi-dimensional. Macroscopically considered, a spatial structure can adopt planar or curved surface forms.

\section{Notions About the Geometry of Dome Shaped Spatial Meshes}

Among the different possibilities for generating by means of nodes, bars and panels a spherical form, what we refer to as the Polyhedrical System has received a vast amount of contributions. Essentially it deals with generating a triangular mesh over the face of a regular or semi-regular polyhedron or over the portion of the sphere that is circumscribed around this polyhedron. The great amount of symmetry planes and rotation symmetry axes present in a geometric body of these characteristics means that the number of different elements in the triangulation (vertices, edges or faces) will be a minimum, as what happens on one of the faces - like face A-D-E in Fig. 2 left - of the regular polyhedron can be reproduced identically on every other face. All of the symmetry planes in a regular polyhedron produce great circles over its circumscribed sphere; the strong influence that these great circles have on the design of the triangular mesh account for the name it receives: Geodesic Dome.

The truth is that the symmetry planes and axis in a regular polyhedron affect every one of its faces, so the proposition stated in the preceding paragraph can be improved: only one part of a regular polyhedron's face requires different elements for its triangulation; the rest propagates around the entire polyhedron or its circumscribed sphere according to its symmetry conditions. In Fig. 2-left the elementary fragment of a regular icosahedron is shaded: 120 of such triangles are reproduced to generate the circumscribed sphere.

The basic model for the generation of a triangulation of the sphere is established through very diverse ways according to the approach taken by the 
different designers. We are proposing in this paper a general approach derived from Coxeter's mosaic [1] of type $\{3,5+\}_{b, c}$ in which the edges of the mosaic do not have to be parallel to the edges of the polyhedron neither the vertices have to belong to the symmetry planes. Figure 2-right shows the development pattern for the $\{3,5+\}_{2,1}$ and $\{3,5+\}_{4,2}$ models. In this way, the pattern of a triangle of $1 / 120$ of the sphere's surface is reproduced all around it.

Figure 1-left shows an example of a spherical dome of the geodesic type. In it the procedure for generating the triangular mesh has followed these rules: (i) Selection of the initial polyhedron (usually a regular icosahedron); (ii) Determination of the triangular network on the polyhedron's face (or to be more precise, on a triangle like A-M-N in Fig. 2-left). The triangular network can be formulated in many diverse ways but Coxeter's notation is valid for all of them, that is, any procedure can be formulated by means of the $\{3,5+\}_{b, c}$ notation; (iii) Projection of this network on the sphere circumscribing the polyhedron. In any of these cases, the design process is aided by numerical calculation methods for obtaining the positions and dimensions of the bars. References [1] and [2] are recommended for a more detailed view of the aforementioned questions.

\section{An Introduction to the Geometry of Plate Meshes. Duality}

Types of spatial meshes like the one shown in Fig. 1-left are named lattice structures (the basic structural element is a bar). As contrasted with the lattice structures, Wester [9] has proposed an interesting alternative suggesting that a plate structure (see Fig. 1-right), where plates (plane elements which are able to resist forces in their own plane only) are stabilized by shear forces, constitutes a new type and concept of structure with an applicability that is, perhaps, deeper than the former.

From the point of view of Statics, lattice and plate designs are equivalent: the equilibrium of force vectors on a node of a lattice structure is formulated in the same way as the equilibrium of moments (created by forces around a reference point) on a face of a plate structure. This equivalence is known as Duality.

From the point of view of Morphology, there can be found a relationship between a polyhedrical approach obtained via lattice and its corresponding one via plate (and vice versa). Wester proposes this correspondence in terms of Duality; three types of dual transformation, DuT, DuM and DiT [8] are, basically, able to supply the mentioned equivalence lattice-plate. It is remarkable that some of these dual transformations give, from a spherical lattice, dual solutions such as double curved shapes different from the sphere (the reciprocal one is possible too).

Besides Wester, some other researchers have studied structural dualism. Concerning the procedure to obtain the shape of the structure, it can be assumed that its geometrical support is the transformation known as polarity. 


\section{Voronoi Diagram and Delaunay Triangulation on the Plane}

The topological similarity between Plate Structures and the Voronoi Diagram cannot pass unnoticed. The same happens when someone considers a lattice mesh (a geodesic dome as Fuller, i.e.) and contemplates the tessellation arising from a Delaunay Triangulation. Computational Geometry can supply some new ideas to the structural design. We will make use of them in the creation of a new way of designing of structural shapes. The most important feature is that the procedure works in 2D space, since a flat Voronoi Diagram will become a polyhedron that approximates a double curved structure (duality guarantees that an inversive transformation applied to a Delaunay Triangulation becomes a lattice grid inscribed into a Sphere).

\section{Some useful properties of the Voronoi diagram.}

Assisted by Fig. 3-left, consider [6] the stereographic projection which describes the restriction of the inversion on $E^{3}$ to the plane $z=1$ (or equivalently, to the sphere $E$, inversive image of this plane). Suppose then a plane $P$ holding $C$ (the center of $E$ ). Whatever the angle that this plane forms with the $O X Y$, it is well known that the inversive shape of the circumference $F_{1}$ (arising from the intersection of $P$ with $E$ ) is another circumference $F^{\prime}{ }_{1}$, the center of which is $C^{\prime}$, resulting from the intersection between the plane $z=1$ and the line perpendicular to $P$ from $O$ (pole of the inversive transformation).
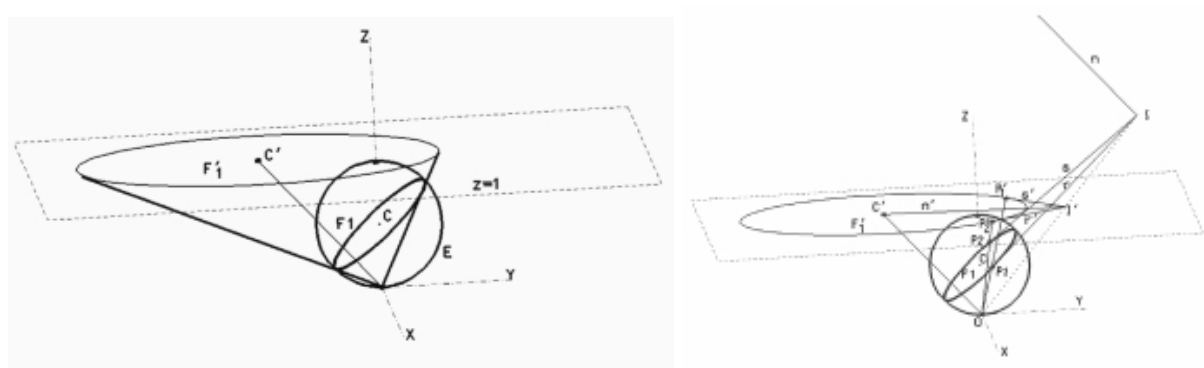

Fig. 3. The projection of $n$ on the plane $z=1$ from $O$ is the perpendicular bisector of the pair of points $P^{\prime}{ }_{1}$ and $P^{\prime}{ }_{2}$ (transformed from $P_{1}$ and $P_{2}$ in the inversion)

Suppose, then, two points $P_{1}$ and $P_{2}$ on the sphere (Fig. 3-right) and consider also the planes $\pi_{1}$ and $\pi_{2}$, tangent to $E$ at the points $P_{1}$ and $P_{2}$. Consider, at last, the line $n$ of intersection of $\pi_{1}$ and $\pi_{2}$.

PROP. 1. The projection of $n$ on the plane $z=1$ from $O$ is the perpendicular bisector of the pair of points $P^{\prime}{ }_{1}$ and $P^{\prime}{ }_{2}$ (transformed from $P_{1}$ and $P_{2}$ in the inversion) 
Let then $S^{\prime}=\left\{P^{\prime}{ }_{1}, P^{\prime}{ }_{2}, \ldots, P^{\prime}{ }_{n}\right\}$ be a set of points in the plane $z=1$. It results that:

PROP. 2. The inversive image of the Voronoi Diagram of $S^{\prime}$ is a polyhedron that approximates the sphere $E\left[x^{2}+y^{2}+(z-1 / 2)^{2}=1 / 4\right]$ in such a way that each one of its faces is tangential to the sphere. There is a symmetric correspondence between each Voronoi polygon and each face of the polyhedron.

These properties are proven in [5].

\section{A 2D Procedure for Creating Meshes Made Up by Non-Triangular Faces}

This last property supposes a more intuitive way for choosing the final shape of a Spatial Mesh (lattice or plate) because its topology can be proposed by means of a $2 \mathrm{D}$ Voronoi Diagram at the plane $z=1$. We can handle different hypotheses easily, as we illustrate in this sample case:

Fig. 4-left: a set of points belonging to the plane $z=1$, (viewed from the point of the infinite of the axis $O Z$ ) is defined. Next, the Voronoi diagram associated with this set is constructed. Fig. 4-right: once property 2 is applied, the polyhedron derived from this diagram is obtained. Fig. 5-left shows the relation between the edges of Voronoi and the edges of the polyhedron: the south pole of the sphere is the center of the inversive transformation.
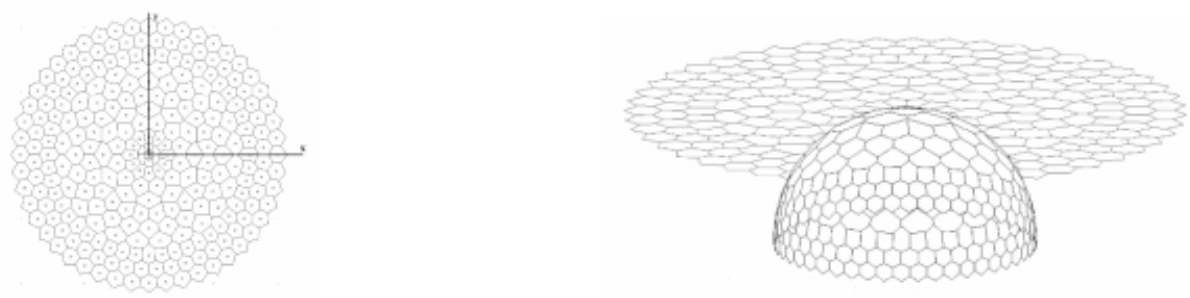

Fig. 4. (left) The inversive image of the Voronoi diagram of $S^{\prime}$ is (right) a polyhedron that approxiamtes the sphere $E\left[x^{2}+y^{2}+(z-1 / 2)^{2}=1 / 4\right]$

Even though the set of points (Fig. 4-left) does not need to follow any rule, we have proposed a distribution by means of concentric rings in order to have different horizontal ones in the sphere: each of them is made up by identical polygons (1 or 2 per ring). A realistic image of the obtained body has been displayed in Fig. 5-right.

\section{A polyhedron approximating a rotation paraboloid}

Suppose $[5,6]$ that we now apply a suitable projective transformation (1), to $E^{3}$ and let $(X, Y, Z, T)$ be the coordinates in the transformed space to the point $(x, y, z, t)$. Specifically, this relation is proposed: 

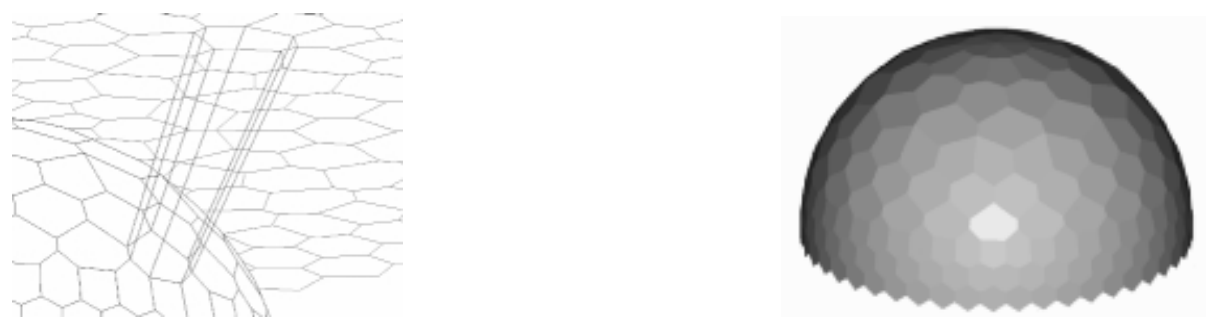

Fig. 5. (left) The relation between the edges of Voronoi and the edges of the spherical polyhedron; (right) a realistic image of the latter

$$
\left(\begin{array}{l}
X \\
Y \\
Z \\
T
\end{array}\right)=\left(\begin{array}{llll}
1 & 0 & 0 & 0 \\
0 & 1 & 0 & 0 \\
0 & 0 & 0 & 1 \\
0 & 0 & 1 & 0
\end{array}\right) \cdot\left(\begin{array}{l}
x \\
y \\
z \\
t
\end{array}\right)
$$

where $t$ and $T$ are, respectively, the homogeneous coordinates of the related points. We denote $M_{H O M}$ to this matrix. Such a transformation:

(i) Is a rotation in $E^{4}$; (ii) Maps the plane $z=0$ to the plane at infinity; (iii) Converts the sphere $E\left[x^{2}+y^{2}+(z-1 / 2)^{2}=1 / 4\right]$ into the rotation paraboloid $P\left[X^{2}+Y^{2}+T^{2}=Z . T\right]$ that, written in inhomogeneous coordinates, results: $Z=X^{2}+Y^{2}+1$; (iv) Maps the plane $z=1$ to the plane $Z=1$; (v) Converts the sphere $E_{2}\left[x^{2}+y^{2}+z^{2}=1\right]$ into a rotation hyperboloid $H_{2}$ that, written in inhomogeneous coordinates, results: $X^{2}+Y^{2}-Z^{2}+1=0$.

Then, if we consider the set of points $S^{\prime}=\left\{P^{\prime}{ }_{1}, P^{\prime}{ }_{2}, \ldots, P^{\prime}{ }_{n}\right\}$ (see point 5 ), on the plane $z=1$, it follows that:

PROP 3. The projection from the point $(0,0,-\infty)$ of the Voronoi Polygons of $S^{\prime}$ to the paraboloid $P\left[Z=X^{2}+Y^{2}+1\right]$ makes up a polyhedron the faces of which are tangent to $\mathrm{P}$. Each point of the set $S^{\prime}$ is transformed to the point of contact between the face of the polyhedron and the surface. The edges of the polyhedron are those transformed from the edges of the Voronoi Diagram of $S^{\prime}$.

Indeed, the transformation keeps all the points of tangency and intersection. So, the same relation proposed in PROP. 2 between the sphere and the plane $z=1$ remains but, now, the pole of projection has gone to the infinite $(0,0,-\infty)$ and the sphere has became a paraboloid.

\section{CR-Tangent Meshes}

This last property relating the edges of the Voronoi Diagram and the edges of the Convex Hull (3D) of the set of generators of a Voronoi Diagram projected on the paraboloid $Z=X^{2}+Y^{2}+1$ is a well known property in Computational Geometry, as proposed in [6] and it has been, in short, the clue needed to reach the proposals shown in point 7 . However, PROP.3 is a particular case of this one: 
PROP 4. The projection from the point $\left(0,0,1 / 2 z_{c}-1\right)$ of the Voronoi Polygons of $S^{\prime}$ to the second order surface $S F\left[X^{2}+Y^{2}-Z^{2}\left(1-2 z_{c}\right)-2 z_{c} Z+1=0\right]$ makes up an approximating polyhedron the faces of which are tangent to $S F$. Each point of set $S^{\prime}$ is transformed to the point of contact between the face of the polyhedron and the surface. The edges of the polyhedron are those transformed from the edges of the Voronoi Diagram of $S^{\prime}$.

- When $z_{c}=1 / 2, S F$ is a rotated paraboloid.

- When $z_{c}<1 / 2, S F$ is a rotated hyperboloid.

- When $z_{c}>1 / 2, S F$ is a rotated ellipsoid.

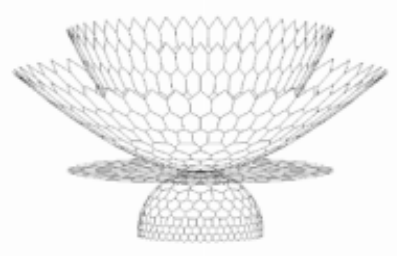

Fig. 6. CR-Tangent models

For the polyhedra approximating the sphere, the paraboloid and the hyperboloid of two sheets represented in Fig. 6, all of them surfaces of revolution, we have proposed the name of CR-TANGENT meshes, CR being the abbreviation of the Spanish term "Cuádricas de Revolución". The term "tangent" is added because the approximating polyhedron is related in this way to a quadric surface (as opposed to lattice meshes in which the approximating polyhedron is inscribed in a sphere).

\section{Matricial Treatment in the Generation of CR-Tangent Meshes: C-Tangent Meshes}

\subsection{Generation of the Revolution Paraboloid}

It is widely known that the equations for an inversion whose center of inversion is the coordinate system's origin and whose inversion Ratio is 1 are the following:

$$
\left(\begin{array}{c}
X^{\prime} \\
Y^{\prime} \\
Z^{\prime} \\
T^{\prime}
\end{array}\right)=\left(\begin{array}{cccc}
\frac{1}{D^{2}} & 0 & 0 & 0 \\
0 & \frac{1}{D^{2}} & 0 & 0 \\
0 & 0 & \frac{1}{D^{2}} & 0 \\
0 & 0 & 0 & 1
\end{array}\right) \cdot\left(\begin{array}{c}
X \\
Y \\
Z \\
T
\end{array}\right)
$$

where 


$$
D^{2}=X^{2}+Y^{2}+Z^{2}
$$

We shall name this matrix $M_{I N V}$. Given a set of points $S^{\prime}=\left\{P^{\prime}{ }_{1}, P^{\prime}{ }_{2}, \ldots, P^{\prime}{ }_{n}\right\}$ lying on the $z=1$ plane, and being $V(S)$ the Voronoi Diagram for such a set, The CR-Tangent mesh of the revolution paraboloid type arises from applying to the vertices of the Diagram the following transformation sequence:

$$
P^{\prime}=M_{H O M} \cdot M_{I N V} \cdot P
$$

Expression whose development is shown below:

$$
\left(\begin{array}{l}
X^{\prime} \\
Y^{\prime} \\
Z^{\prime} \\
T^{\prime}
\end{array}\right)=\left(\begin{array}{llll}
1 & 0 & 0 & 0 \\
0 & 1 & 0 & 0 \\
0 & 0 & 0 & 1 \\
0 & 0 & 1 & 0
\end{array}\right)\left(\begin{array}{cccc}
\frac{1}{D^{2}} & 0 & 0 & 0 \\
0 & \frac{1}{D^{2}} & 0 & 0 \\
0 & 0 & \frac{1}{D^{2}} & 0 \\
0 & 0 & 0 & 1
\end{array}\right) \cdot\left(\begin{array}{c}
X \\
Y \\
Z \\
T
\end{array}\right)
$$

\subsection{Generation of Other Quadric Surfaces of Revolution}

The possibility for the generation of quadric surfaces of revolution other than the paraboloid arises from the fact that a projective transformation (1), being its matrix $M_{H O M}$, can be applied to the polyhedron approximating a sphere which is tangent to the $z=1$ plane and whose center is on the $Z$ axis but having a radius $r$ different of $1 / 2$. Let's suppose that, as it is shown there, the center of the sphere tangent to plane $z=1$ is at the point $\left(0,0, Z_{c}\right)$.

We can reduce these cases to the preceding one if we produce two axis-wise transformations:

I. A translation to the south pole of the circumference $\left(0,0,2 Z_{c}-1\right)$. We shall name this translation matrix $M_{T R A}$.

II. A homothecy being its center the coordinate system's origin (which by now is on the sphere's south pole) and with a ratio of $1 / 2 r$, that will transform the sphere of radius $r=1-Z_{c}$ into one of radius $1 / 2$ and return the generating points for the Voronoi Diagram to the $z=1$ plane. We shall name this transformation matrix $M_{E S C}$. Once applied this two transformations, the problem is reduced to the one stated in the previous point; then the expression (5) applied to the Voronoi Diagram vertices will yield as a result the polyhedron which approximates a sphere of radius $1 / 2$ and then it will only be necessary to undo transformations I and II, which we attain by applying the operative matrices which we shall identify as $M_{E S C(-)}$ y $M_{T R A(-)}$. The result obtained can be summarized as:

$$
P^{\prime}=\left[M_{T R A(-)} \cdot M_{E S C(-)} \cdot M_{I N V} \cdot M_{E S C} \cdot M_{T R A}\right] \cdot P
$$

that can be developed as: 


$$
\left(\begin{array}{l}
X^{\prime} \\
Y^{\prime} \\
Z^{\prime} \\
T^{\prime}
\end{array}\right)=\left(\begin{array}{llll}
1 & 0 & 0 & 0 \\
0 & 1 & 0 & 0 \\
0 & 0 & 1 & S \\
0 & 0 & 0 & 1
\end{array}\right)\left(\begin{array}{cccc}
2 r & 0 & 0 & 0 \\
0 & 2 r & 0 & 0 \\
0 & 0 & 2 r & 0 \\
0 & 0 & 0 & 0
\end{array}\right) \cdot M_{I N V} \cdot\left(\begin{array}{cccc}
\frac{1}{2 r} & 0 & 0 & 0 \\
0 & \frac{1}{2 r} & 0 & 0 \\
0 & 0 & \frac{1}{2 r} & 0 \\
0 & 0 & 0 & 1
\end{array}\right)\left(\begin{array}{cccc}
1 & 0 & 0 & 0 \\
0 & 1 & 0 & 0 \\
0 & 0 & 1 & -S \\
0 & 0 & 0 & 1
\end{array}\right)\left(\begin{array}{c}
X \\
Y \\
Z \\
T
\end{array}\right)
$$

where $S=2 Z_{c}-1$; thus, now the homology transformation is applied:

$$
\left(\begin{array}{l}
X^{\prime \prime} \\
Y^{\prime \prime} \\
Z^{\prime \prime} \\
T^{\prime \prime}
\end{array}\right)=\left(\begin{array}{llll}
1 & 0 & 0 & 0 \\
0 & 1 & 0 & 0 \\
0 & 0 & 0 & 1 \\
0 & 0 & 1 & 0
\end{array}\right) \cdot\left(\begin{array}{l}
X^{\prime} \\
Y^{\prime} \\
Z^{\prime} \\
T^{\prime}
\end{array}\right)
$$

Points $P^{\prime \prime}$ are the vertices of the polyhedron approximating a quadric surface of revolution different from the paraboloid.

\subsection{Attainment of C-Tangent Meshes}

We shall name as C-TANGENT meshes (the name in this case referring to polyhedra which are tangent to quadric surfaces in general, including but not limited to those obtained by revolution) those obtained by applying the homology transformation to a sphere tangent to plane $\mathrm{z}=1$ but placed in any position in space. This added degree of freedom means, as regarding the case studied in the previous point, simply that the center of the sphere can be placed in point $\left(X_{c} Y_{c} Z_{c}\right)$, always maintaining the condition that $r=1-z_{c}$. Summarising, the following transformation expressions result:

$$
\left(\begin{array}{l}
X^{\prime} \\
Y^{\prime} \\
Z^{\prime} \\
T^{\prime}
\end{array}\right)=\left(\begin{array}{llll}
1 & 0 & 0 & X_{c} \\
0 & 1 & 0 & Y_{c} \\
0 & 0 & 1 & S \\
0 & 0 & 0 & 1
\end{array}\right)\left(\begin{array}{cccc}
2 r & 0 & 0 & 0 \\
0 & 2 r & 0 & 0 \\
0 & 0 & 2 r & 0 \\
0 & 0 & 0 & 0
\end{array}\right) \cdot M_{I N V} \cdot\left(\begin{array}{cccc}
\frac{1}{2 r} & 0 & 0 & 0 \\
0 & \frac{1}{2 r} & 0 & 0 \\
0 & 0 & \frac{1}{2 r} & 0 \\
0 & 0 & 0 & 1
\end{array}\right)\left(\begin{array}{cccc}
1 & 0 & 0 & -X_{c} \\
0 & 1 & 0 & -Y_{c} \\
0 & 0 & 1 & -S \\
0 & 0 & 0 & 1
\end{array}\right)\left(\begin{array}{c}
X \\
Y \\
Z \\
T
\end{array}\right)
$$
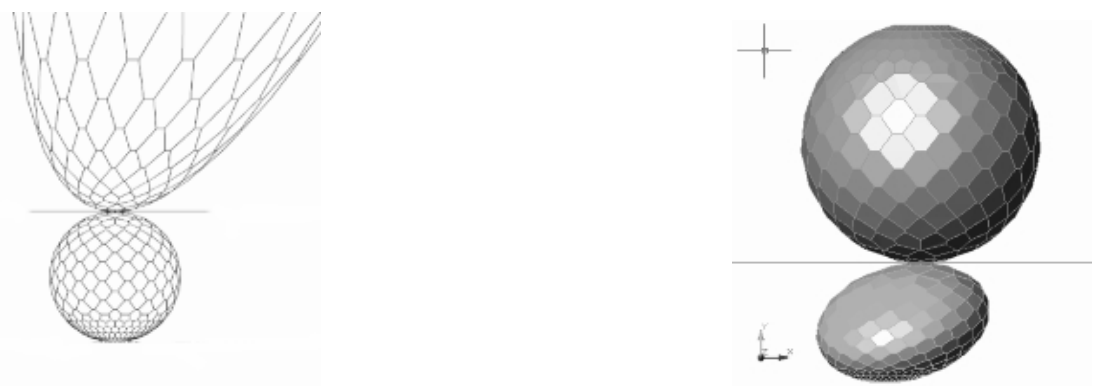

Fig. 7. C-Tangent models: (left) paraboloid; (right) ellipsoid 
Points $P^{\prime \prime}$, obtained by (8), are the vertices for the polyhedron approximating a quadric surface. It can be easily demonstrated that the two preceding points present only special cases of this expression. Two of the surfaces obtained this way are shown in Fig. 7.

\section{Summarising}

Properties and methods of Computational Geometry lead to a simple 2D procedure, with a very simple computer implementation, that can be used for generating new Spatial Structure models (of the Plate type, see point 3) shaped as rotated ellipsoid, paraboloid or hyperboloid. The approximating polyhedron holds non-triangular polygons tangent to the surface it approaches. It is not difficult to notice that, following the procedure described, local changes in this resulting polyhedron are really easy to carry out. On the other hand, the properties of the Voronoi Diagram guarantee the structural stability of the body obtained.

Following a similar way as the one we have shown in this paper it is very easy to obtain the lattice type of structure transforming a Delaunay Triangulation instead of the Voronoi Diagram. We remark again that: our design effort is to select the most adequate simple set of points on the plane, whose associated tessellation (Voronoi Diagram o Delaunay triangulation) provides the basis for the generation of a Spatial Dome, making it possible to select a lattice type or a plate one. Wherever these points are placed, we find a polyhedron as the solution; as designers, we need only to study the position of the generators (or locally move some of them) in order to obtain a "good design".

\section{References}

1. Coxeter, H.S.M.: Regular Complex Polytopes Cambridge University Press, London (1974) 9-11

2. Critchlow, K.: Order in Space, Thames and Hudson, Norwich (1969) 76

3. Makowski, Z. S.: Analysis, Design and Construction of Braced Domes. Cambridge University Press, Cambridge (1984)

4. Makowski, Z. S.: Steel Space Structures. Verlag Stahleisen, Dusseldorf (1968)

5. Otero, C. Gil, V., Alvaro, J.I.: CR-Tangent meshes. International Association for Shell and Spatial Structures Journal 21(132) (2000) 41-48

6. Preparata, F., Shamos, I.: Computational Geometry: An Introduction. SpringerVerlag, New York (1985) 244-247

7. Tsuboi, Y.: Analysis, design and realization of space frames. (Working Group of Spatial Steel Structures), International Association for Shell and Spatial Structures Bulletin 84 (1984) 11-30

8. Wester, T.: A geodesic dome-type based on pure plate action. International Journal of Space Structures 5(3-4) (1990) 155-167

9. Wester, T.: The Structural Morphology of Basic Polyhedra. John Wiley and Sons, New York (1997) 301-342 